\title{
SIGNIFICADO DE LA MEDIA, MEDIANA Y MODA EN TEXTOS ESCOLARES DE SÉPTIMO BÁSICO
}

\author{
MEANING OF THE MEAN, MEDIAN AND MODE IN SEVENTH \\ GRADE SCHOOL TEXTS
}

Jaime I. García-García

jaime.garcia@ulagos.cl

Universidad de Los Lagos,

Osorno, Chile

Sebastián H. Vásquez Chicao

sebastianhvasquez@gmail.com

Universidad de Los Lagos, Osorno, Chile

Liceo San Sebastián, Osorno, Chile
Ingrid B. Urrutia Leiva

urrutiaingrid79@gmail.com

Universidad de Los Lagos, Osorno, Chile

Colegio Bicentenario Crucero "Oscar Daniel", Río Bueno, Chile

Elizabeth Hernández Arredondo

elizabeth.hernandez@ulagos.cl

Universidad de Los Lagos, Osorno, Chile

\section{RESUMEN}

En este trabajo se presenta un estudio del significado de la media, mediana y moda en una muestra de dos textos escolares de séptimo año de Educación Básica de Chile. Para el desarrollo del estudio, hemos considerado algunas herramientas de análisis del Enfoque Ontosemiótico del Conocimiento y la Instrucción Matemáticos, identificando la presencia de cada elemento del significado (situaciones-problema, procedimientos, conceptosdefinición, propiedades, elementos lingüísticos y argumentos) de la media, mediana y moda en los textos escolares. Los resultados evidencian la ausencia de diversos elementos en la presentación de la media, mediana y moda en los textos escolares chilenos de séptimo básico; en consecuencia, se deducen algunas sugerencias de los elementos de estas medidas de tendencia central que consideramos necesarios para su incorporación en las aulas para lograr su enseñanza más significativa.

\section{PALABRAS CLAVE:}

Enfoque ontosemiótico, Medidas de tendencia central, Educación Básica, Libros de texto.

\begin{abstract}
This paper presents a study of the meaning of the mean, median and mode in a sample of two seventh-year textbooks of Basic Education in Chile. For the development of the study, we have considered some analysis tools of the Onto-semiotic Approach to Mathematical Cognition and Instruction, identifying the presence of each element of meaning (problem-situations, procedures, concepts-definitions, properties, linguistic elements and arguments) of the mean, median and mode in the school texts. The results show the absence of various elements in the presentation of the mean, median and mode in Chilean school textbooks for the seventh grade; consequently, some suggestions are deduced from the elements of these measures of central tendency that we consider necessary for their incorporation in the classrooms to achieve their most meaningful teaching.
\end{abstract}

\section{KEYWORDS:}

Onto-semiotic Approach, central tendency measures, primary education, textbooks. 


\section{Introducción}

Hace más de 25 años, la Estadística se ha incluido paulatinamente en el currículo de matemáticas de diversos países (Vásquez y Alsina, 2014) con el fin de desarrollar en el estudiante competencias para la recolección y organización de datos; la construcción, lectura e interpretación crítica de tablas y gráficos estadísticos; el cálculo e interpretación de medidas estadísticas; el análisis de la variabilidad; la predicción y toma de decisiones con base en los datos; entre otras. En general, para desarrollar la alfabetización estadística en el estudiante.

La alfabetización estadística, en términos de Gal (2004), se refiere a la capacidad de las personas para interpretar y evaluar críticamente información estadística, argumentos relacionados con los datos o fenómenos estocásticos, que pueden encontrar en diversos contextos; y la capacidad para discutir o comunicar sus opiniones respecto a dicha información, cuando sea relevante.

Del Pino y Estrella (2012) señalan que un ciudadano alfabetizado estadísticamente debería ser capaz de leer e interpretar representaciones estadísticas (tablas y gráficos) y medidas de resumen que se presentan en los medios de comunicación. En ese sentido, las medidas de tendencia central (MTC), parte de las medidas de resumen, son consideradas como elementos de la alfabetización estadística que todo estudiante debería aprender y comprender durante su educación.

Con respecto a los estudios sobre las MTC (media, mediana y moda), podemos señalar dos líneas de investigación: la primera enfocada en los errores y dificultades en la comprensión del significado de las MTC por parte de estudiantes y profesores de diversos niveles educativos (p. ej., Batanero, 2000; Cobo, 2003; Del Puerto et al., 2007; Escobedo y Mayén, 2018; Estrella, 2016; Mayén, 2009; Mayén et al., 2007); y la segunda centrada en el análisis de las MTC en libros de texto (p. ej., Cabrera, 2014; Carvalho y Gitirana, 2014; Cobo, 2003; Cobo y Batanero, 2004; Díaz-Levicoy et al., 2020; Estrella, 2008; Mayén, 2009; Ocoró y Ocoró, 2016).

Las investigaciones en torno al análisis del libro de texto radican en el reconocimiento de la importancia que tiene este recurso pedagógico en el proceso de enseñanza-aprendizaje de las matemáticas en las aulas escolares producto de una transposición didáctica (Chevallard, 1991), debido a que es la herramienta didáctica más usada por el profesor e influye en el aprendizaje de los estudiantes, así como su relación directa con la implementación del currículo (Herbel, 2007) y su contribución al éxito del cumplimiento y puesta en práctica de las directrices curriculares (Cantoral et al., 2015).

Por lo anterior, este estudio tiene como objetivo analizar el significado de la media, mediana y moda en una muestra de dos textos escolares chilenos de séptimo año de Educación Básica.

\section{Referentes teóricos}

\subsection{El enfoque ontosemiótico (EOS)}

Para el desarrollo de este estudio hemos utilizado algunas herramientas de análisis del modelo teórico denominado Enfoque Ontosemiótico (EOS) del Conocimiento y la Instrucción Matemáticos, propuesto por Godino y sus colaboradores (Godino et al., 2007, 2020).

El EOS es un modelo teórico que articula diversas perspectivas de las disciplinas y dimensiones relacionadas con el aprendizaje y enseñanza de las matemáticas para poder abordar problemas de tipo epistemológico, ontológico, semiótico-cognitivo y educativo (Pino-Fan, 2013). En el EOS se reconoce una doble naturaleza para las matemáticas: como sistema de objetos y como sistema de prácticas. La noción de práctica matemática se entiende como "toda actuación o expresión (verbal, gráfica, etc.) realizada por alguien para resolver problemas matemáticos, comunicar a otros la solución obtenida, validarla o generalizarla a otros contextos y problemas" (Godino y Batanero, 1994, p. 334); y la de objeto matemático como cualquier entidad material o inmaterial que interviene en la práctica matemática, apoyando y regulando su realización (Font et al., 2013).

La realización de una práctica matemática pone en funcionamiento conocimiento matemático. Este conocimiento se expresa en un lenguaje, que se puede descomponer en lenguaje verbal y lenguaje simbólico o gráfico. Los enunciados lingüísticos derivados de las prácticas matemáticas son, a su vez, la expresión de conceptos, proposiciones y procedimientos, y con base en estos, se constituyen argumentos que dan cuenta de la validez de las acciones.

De acuerdo con Font et al. (2007), de esta práctica matemática emergen seis tipos de objetos matemáticos primarios, denominados "elementos del significado", que corresponden a:

a) Situaciones-problema: referentes a aplicaciones extra matemáticas, tareas, ejercicios, cuestionamientos, entre otros, asociados al objeto matemático, en nuestro caso la media, mediana y moda.

b) Procedimientos: referentes a algoritmos, operaciones, técnicas de cálculo, entre otros, que se realizan en los diversos tipos de prácticas.

c) Conceptos-definición: introducidos mediante definiciones o descripciones, explícitas 0 implícitas, del objeto matemático (media, mediana, ...).

d) Propiedades/proposiciones: enunciados (declaraciones) sobre conceptos (postulados, teoremas, etc.). 
e) Elementos lingüísticos: corresponden a términos, expresiones, notaciones, tablas, gráficos, etc., en sus diversos registros (oral, escrito, ...).

f) Argumentos: corresponden a enunciados (discursos) usados para probar o explicar las proposiciones y procedimientos, deductivos o de otro tipo; demostraciones que utilizamos para validar propiedades.

Estos elementos de significado están relacionados entre sí, formando configuraciones de redes de objetos intervinientes y emergentes de los sistemas de prácticas (Godino et al., 2007). De acuerdo con los autores, un sujeto comprende un determinado objeto matemático cuando lo usa de manera idónea en diferentes prácticas. Por ello, para el análisis de las MTC en los textos escolares de séptimo básico se considerará la tipología de objetos matemáticos primarios para identificar la presencia de cada elemento del significado de la media, mediana y moda.

\subsection{Las medidas de tendencia central en las Bases Curriculares de Chile}

El Ministerio de Educación de Chile (MINEDUC), en las Bases Curriculares de Educación Básica (MINEDUC, 2016, 2018) establece Objetivos de Aprendizaje (OA) que precisan la expectativa formativa que se espera que logren los estudiantes en cada asignatura, por cada año escolar. A partir de una revisión en estas bases, identificamos los OA que declaran el estudio de las MTC (Tabla 1).

Tabla 1. Objetivos de aprendizaje $(\mathrm{OA})$ relacionados con las MTC en Educación Básica.

Nota. Elaboración propia a partir de MINEDUC $(2016,2018)$.

\begin{tabular}{|l|l|l|}
\hline $\begin{array}{l}\text { Grado } \\
\text { escolar }\end{array}$ & $\begin{array}{l}\text { Rango } \\
\text { etario }\end{array}$ & Objetivo de aprendizaje \\
\hline Quinto & 10-11 años & $\begin{array}{l}\text { 23. Calcular el promedio de } \\
\text { datos e interpretarlo en su } \\
\text { contexto. (MINEDUC, 2018, p. } \\
\text { 249) }\end{array}$ \\
\hline Séptimo & 12-13 años & $\begin{array}{l}\text { 17. Mostrar que comprenden } \\
\text { las medidas de tendencia } \\
\text { central y el rango: } \\
\text { - Determinando las medidas } \\
\text { de tendencia central para } \\
\text { realizar inferencias sobre la } \\
\text { población. } \\
\text { - Determinando la medida de } \\
\text { tendencia central adecuada } \\
\text { para responder un problema } \\
\text { planteado. } \\
\text { - Utilizándolos para comparar } \\
\text { dos poblaciones. } \\
\text { - Determinando el efecto de } \\
\text { un dato que es muy diferente } \\
\text { a los otros. (MINEDUC, 2016, } \\
\text { p. 109) }\end{array}$ \\
\hline
\end{tabular}

Como podemos observar, el estudio de las tres MTC se declara en séptimo básico; por ello, enfocamos el análisis en los textos escolares de dicho grado escolar.

\section{Algunas investigaciones sobre análisis de las MTC en libros de textos}

A continuación, se presentan algunas investigaciones centradas en el análisis de los elementos del significado de la media, mediana y moda en libros de texto, con el propósito de situar nuestro estudio.

En Cobo (2003) se presenta un estudio del significado institucional de las MTC en una muestra de 22 libros de texto de tercer (14-15 años) y cuarto (15-16 años) grado de Educación Secundaria Obligatoria (ESO) de España. Se analiza el tipo de problemas, algoritmos de cálculo, definiciones, propiedades, representaciones y argumentos, identificando aquellos elementos de significado más comunes presentados en la muestra de libros sobre las MTC, por ejemplo, obtener un elemento representativo de un conjunto de valores dados cuya distribución es aproximadamente simétrica, o bien, obtener un valor representativo de una colección de datos en situaciones en las que lo que interesa fundamentalmente es el valor dominante del conjunto. Con respecto al trabajo de las MTC, la autora señala que este le da mucha más importancia a la memorización de las definiciones y al cálculo de la media, mediana y moda, que al estudio de sus propiedades. Por su parte, Mayén (2009) analiza los elementos del significado de las MTC en dos libros de texto y un cuaderno de prácticas de matemáticas de tercer grado (14-15 años) de Educación Secundaria de México. Sus resultados concuerdan con los reportados por Cobo (2003), por ejemplo, en relación con los procedimientos más frecuentes (cálculo de la media, la mediana y la moda de una variable discreta con datos aislados), así como la escasez de aquellos de mayor complejidad tanto para la media como para la mediana.

Carvalho y Gitirana (2014) analizan los significados, propiedades y representaciones de la media aritmética en libros de texto de Brasil. Sus resultados evidencian que la mayoría de las actividades no promueven la reflexión de las propiedades; el significado con mayor presencia es la media como elemento representativo de un conjunto de datos, y la representación más utilizada es la lengua materna (lenguaje), considerada en el enunciado escrito, muchas veces ayudada con fórmulas y símbolos inherentes a la dimensión matemática de la media aritmética. En otra investigación, Ocoró y Ocoró (2016) analizan la forma en que se presentan las MTC en dos libros de texto colombianos de séptimo grado. Sus resultados evidencian que no se presentan todas las propiedades de cada MTC, no se trabaja la media ponderada, carecen de ejemplos e ilustraciones asociadas a sus actividades, y no ofrecen ejercicios de mayor dificultad que permitan al estudiante interiorizarse en estas medidas. 
En el contexto chileno, Estrella (2008) analiza la transposición didáctica de las MTC en un libro de texto de séptimo básico, es decir, las transformaciones que se producen en los conocimientos sobre las MTC desde un texto de nivel universitario para ser enseñados en dicho grado. Sus resultados muestran que el libro de texto presenta imprecisiones con respecto a los términos y sus significados, las MTC son presentadas a través de fórmulas y las tareas se enfocan únicamente a su uso.

Con relación a lo anterior, se observa que son escasas las investigaciones enfocadas en el análisis de la media, mediana y moda, de manera conjunta, en los libros de textos. Bajo esta perspectiva, nuestro estudio aporta resultados de los elementos del significado de las MTC que se presentan en los textos escolares y se trabajan en las aulas chilenas de séptimo básico.

\section{Metodología}

Nuestro estudio se enmarca en una metodología cualitativa (Pérez-Serrano, 1994), de tipo descriptiva (Hernández et al., 2010), y se usa como método de investigación el análisis de contenido (LópezNoguero, 2002), debido a que el objetivo es analizar cada elemento del significado (situaciones-problema, procedimientos, conceptos-definición, propiedades, elementos lingüísticos y argumentos) de la media, mediana y moda en los textos escolares de séptimo año de Educación Básica. La muestra seleccionada, no probabilística, es intencional y está formada por el texto del estudiante (TE) y el cuaderno de actividades (CA) de séptimo básico; ambos textos escolares siguen el marco curricular actual (MINEDUC, 2016) y son distribuidos gratuitamente para todos los centros educativos particulares y públicos con carácter obligatorio por parte del Ministerio de Educación de Chile (MINEDUC). En este sentido, estos textos escolares fueron elegidos por su amplio uso en las aulas chilenas.

Tabla 2. Textos escolares utilizados en el análisis.

Nota. Elaboración propia.

\begin{tabular}{|c|c|}
\hline Texto escolar & Referencia \\
\hline TE & $\begin{array}{l}\text { Iturra, F., Manosalva, C., Romero, } \\
\text { D., y Ramírez, M. (2020). } \\
\text { Matemática. } 7^{\circ} \text { Básico. Texto del } \\
\text { estudiante. SM S.A. }\end{array}$ \\
\hline CA & $\begin{array}{l}\text { Arce, D. (2020). Matemática. } 7^{\circ} \\
\text { Básico. Cuaderno de actividades. } \\
\text { SM S.A. }\end{array}$ \\
\hline
\end{tabular}

Para el análisis de los textos escolares seguimos el método propuesto por Cobo (2003): 1) lectura rigurosa de la Lección 17, Medidas de Tendencia Central, de cada texto escolar, con el propósito de comparar el contenido con cada elemento del significado de la media, mediana y moda, y con ello determinar su presencia (en caso de encontrar alguno nuevo se describe); 2) selección de imágenes para ejemplificar cada elemento del significado encontrado; 3) realización de un resumen escrito en el que se analizan los elementos identificados; 4) elaboración de tablas que resuman los resultados, y a su vez, obtención de conclusiones sobre los elementos del significado de las MTC en los textos escolares utilizados por los estudiantes de séptimo básico. Cabe señalar que el primer paso siguió un proceso sistemático y cíclico, cuya fiabilidad se aseguró mediante la comparación de los resultados del análisis realizado por cada uno de los autores, y en caso de desacuerdo, se realizó un nuevo proceso de análisis hasta llegar a un consenso. Para el análisis de contenido se consideró cada elemento del significado (situaciones-problema, procedimientos, conceptos-definición, propiedades, elementos lingüísticos y argumentos) de las MTC, a partir de la descripción de cada uno de ellos propuestos por Cobo (2003) y Mayén (2009), los cuales se describen y ejemplifican a detalle en el apartado de resultados.

a) Situaciones-problema (SP). a) Media (M): SPM1 [situación-problema asociada a la media, uno]. Estimar una medida a partir de diversas mediciones realizadas, en presencia de errores. SPM2. Obtener una cantidad equitativa al hacer un reparto para conseguir una distribución uniforme. SPM3. Obtener un elemento representativo de un conjunto de valores dados cuya distribución es aproximadamente simétrica. SPM4. Conocer el valor que se obtendrá con mayor probabilidad al tomar un elemento al azar de una población para una variable con distribución aproximadamente simétrica. SPM5. Comparación de dos distribuciones de datos con variables numéricas. b) Mediana (ME): SPME1. Encontrar un resumen estadístico de posición central, en situaciones en las que la media no es suficientemente representativa. SPME2. Encontrar un resumen estadístico de posición central para variables ordinales. SPME3. Efectuar comparaciones de dos o más colecciones de datos usando gráficos de caja. c) Moda (MO): SPMO1. Obtener como valor representativo de una colección de datos en situaciones en las que lo que interesa fundamentalmente es el valor dominante del conjunto. SPMO2. Encontrar el valor representativo en datos cualitativos.

b) Procedimientos (P). a) Media (M): PM1 [procedimiento asociado a la media, uno]. Cálculo de la media de una variable discreta con datos aislados. PM2. Cálculo de la media de una variable discreta con datos presentados en tablas de frecuencias. PM3. Cálculo de la media de una variable continua o discreta con datos agrupados en intervalos de clases. PM4. Cálculo gráfico. PM5. Cálculo con calculadora u ordenador. PM6. Inversión del algoritmo de cálculo de la media. PM7. Construir una distribución de media dada. b) Mediana (ME): PME1-PME2. Cálculo de la mediana con datos no agrupados en clases, número de datos impar y par, respectivamente. PME3-PME4. 
Cálculo de la mediana a partir datos presentados en una tabla de frecuencias; casos de un número par e impar de valores, respectivamente. PME5. Cálculo de la mediana a partir de datos agrupados en clases. PME6. Cálculo de la mediana a partir de datos presentados en un gráfico. c) Moda (MO): PM01. Cálculo de la moda en una variable discreta con datos aislados. PMO2. Cálculo de la moda en una variable discreta con datos presentados en una tabla de frecuencias. PMO3. Cálculo de la moda de una variable discreta o continua con datos agrupados en intervalos de clase. PMO4. Cálculo a partir de un gráfico.

c) Concepto-definición (CD). a) Media (M): CDM1 [concepto-definición asociado a la media, uno]. La definición de la media como la suma ponderada de cada uno de los valores de la variable, multiplicado por su frecuencia. CDM2. Definición de media, como promedio aritmético de un conjunto de datos. b) Mediana (ME): CDME1. Mediana como centro de la distribución. CDME2. La mediana es el valor de la variable estadística que divide en dos efectivos iguales. CDME3. El valor de la variable estadística tal que la ordenada del diagrama acumulativo de frecuencias absolutas es igual a n/2. CDME4. La mediana como el valor de la variable estadística tal que la ordenada de la curva de distribución empírica es igual a 1/2. c) Moda (MO): CDMO1. La moda es el valor más frecuente de la variable estadística. CDMO2. La moda es el valor que corresponde al máximo del diagrama de barras o histograma.

d) Propiedades (P). a) Numéricas (N): PN1 [propiedad numérica, uno]. La media, la mediana y la moda de un conjunto de datos son siempre valores pertenecientes al rango de la variable. PN2. La mediana y la media pueden no coincidir con ninguno de los valores de los datos, mientras que la moda siempre es uno de estos valores. PN3. En el cálculo de la media y la moda intervienen todos los valores de los datos, no así en el caso de la mediana. PN4. La moda y la mediana son, en ocasiones, invariantes si cambian algunos de los datos, mientras que la media sí se ve afectada por cualquier cambio en los datos. b) Algebraicas (A): PA1. Operación interna. Esta propiedad solo es válida para la moda, debido a que conserva su valor en el conjunto de datos, mientras que el cálculo de la media y la mediana no, ya que pueden tomar un valor distinto a todos sus elementos. PA2. La media, mediana y moda, consideradas como operación algebraica, no tienen elemento neutro ni simétrico. PA3. No tienen la propiedad asociativa. PA4. Son conmutativas. PA5. Las medidas de tendencia central conservan los cambios de origen y escala. PA6. La media de la suma de dos o más variables es la suma de las medias. En el caso de la mediana y la moda no se cumple. PA7. La moda puede no existir o, si existe, no ser única, mientras que la media y la mediana siempre existen. c) Estadísticas (E): PE1.
La media, mediana y moda son representantes de un colectivo. PE2. La media coincide con el centro de gravedad del conjunto de datos. PE3. En distribuciones simétricas, la mediana, la media y la moda coinciden. PE4. La media es un estadístico menos resistente que la mediana y la moda. PE5. La suma de las desviaciones de un conjunto de datos con respecto a su media es cero. PE6. Es respecto a la media cuando la suma de los cuadrados de las desviaciones es mínima. PE7. Es preferible a la mediana en distribuciones con datos agrupados en los que al menos un intervalo es abierto. PE8. Existe moda/s para variables cuantitativas y cualitativas. PE9. En distribuciones con más de una moda, la mediana es el mejor representante del conjunto de datos.

e) Elementos lingüísticos. Términos, símbolos, tablas y gráficos.

f) Argumentos (ARG). ARG1 [argumento, uno]. Comprobación de casos particulares y contraejemplos. ARG2. Uso de gráficos como justificación. ARG3. Razonamientos algebraicos. ARG4. Razonamientos verbales deductivos.

\section{Resultados}

A continuación, se presentan los resultados del análisis de los elementos del significado de la media, mediana y moda presentes en los textos escolares chilenos de séptimo básico. Para esto, se muestran algunas imágenes para ejemplificar los elementos encontrados, se incluyen tablas que resumen los resultados, y se obtienen conclusiones sobre los hallazgos.

\subsection{Situaciones-problema}

En primer lugar, se analizan las situaciones-problema asociadas a cada MTC. La SPM3 se observa en la actividad de la Figura 1, donde se solicita obtener la media de las estaturas de cada conjunto de datos cuya distribución es aproximadamente simétrica.

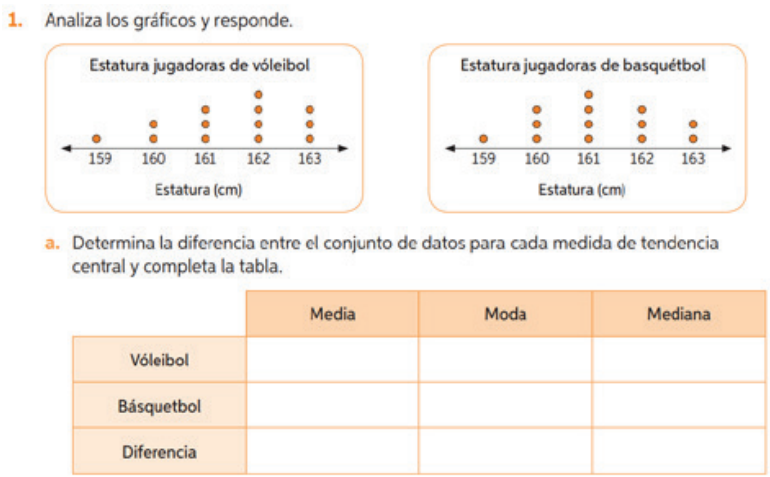

Figura 1. Situación-problema 3 asociada a la media Nota. CA, p. 114 
En el inciso d) de la actividad de la Figura 2 se presenta la SPM5, donde la media aparece como MTC adecuada para comparar los dos conjuntos de calificaciones, en condiciones similares.

6. A continuación, se presentan las calificaciones de Alan en dos asignaturas. Inglés: 5,6 - 5,7 - 6,0 - 6,1 - 5,8 - 6,2 - 6,2

Ciencias Naturales: 4,3-5,7 -6,3-6,5 - 7,0 - 5,9 - 5,9

Calcula el rango de cada asignatura. ¿Qué puedes decir de los valores obtenidos?

b. Calcula el promedio de Alan en ambas asignaturas. ¿Qué significan esos valores?

c. Construye un gráfico de lineas en el que muestres el rendimiento de Alan en ambas asignaturas.

d. Dibuja la media en el gráfico. ¿En qué asignatura crees que la media es más representativa? Explica.

Figura 2. Situación-problema 5 asociada a la media Nota. TE, p. 201

En este estudio hemos considerado una nueva situación-problema asociada a la media, SPM6, donde el cálculo de la media aparece como tarea de tipo procedimental; esta se observa en la actividad de la Figura 3.

3. Calcula la media aritmética y el rango de los siguientes datos:

a. $158,160,168,156,166,158,160,168,160,168,158,156,164,162,166,164,168$, $160,162,162,158,156,166,160,168$

Figura 3. Situación-problema 6 asociada a la media Nota. CA, p. 111

En el inciso b) de la actividad de la Figura 4 se observa la SPME1, donde la mediana se presenta como la MTC más apropiada, ya que no se vería afectada por agregar un dato con valor extremo.

1. Para unas olimpiadas de Matemática los profesores han escogido 15 jóvenes que representarán al colegio. Las edades de los competidores son:

$14,11,10,15,12,15,10,16,10,10,11,14,15,16,10$.

¿Qué edad tiene como máximo la mitad más joven de los competidores?

a. Ordena los datos en forma creciente y determina el término central.

b. La respuesta anterior corresponde a la mediana. ¿Qué sucedería con la mediana si al grupo se agrega un joven de 15 años?

Figura 4. Situación-problema 1 asociada a la mediana Nota. TE, p. 204.

La SPME3 se observa en la Figura 5, donde la mediana es la MTC más significativa para comparar los datos de cada grupo de edades.
1. Analiza el paso a paso y observa el ejercicio resuelto. Luego, realiza las actividades propuestas.

El departamento de deportes de un municipio inició las inscripciones para el taller de zumba. Cada inscrito tiene derecho a asistir a una clase semanal las cuales se imparten los lunes (grupo 1) o los miércoles (grupo 2). Para conocer el impacto de este taller, la profesora registró las edades (años) de los asistentes durante la primera semana.

¿Cómo es la distribución de las edades en cada uno de los grupos?

Paso 1: Identifica las caracteristicas de los datos de cada grupo.

\begin{tabular}{|l|c|c|c|c|c|c|}
\hline \multicolumn{1}{|c|}{ Dia } & Asistentes & $\begin{array}{c}\text { Edad } \\
\text { promedio }\end{array}$ & Mediana & $\begin{array}{c}\text { Edad } \\
\text { minima }\end{array}$ & $\begin{array}{c}\text { Edad } \\
\text { máxima }\end{array}$ & Rango \\
\hline Lunes & 11 & 25 & 16 & 14 & 45 & 31 \\
\hline Miércoles & 11 & 25 & 27 & 21 & 28 & 7 \\
\hline
\end{tabular}

Figura 5. Situación-problema 3 asociada a la mediana Nota. TE, p. 201 Nota. TE, p. 206.

En este estudio hemos considerado una nueva situación-problema asociada a la mediana, SPME4, donde el cálculo de la mediana aparece como tarea de tipo procedimental; esta se observa en los tres incisos de la actividad de la Figura 6.

1. Calcula la mediana de cada conjunto de datos.

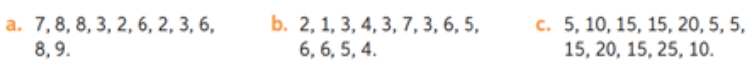

Figura 6. Situación-problema 4 asociada a la mediana Nota. CA, p. 113

También, en este estudio hemos considerado una nueva situación-problema asociada a la mediana, SPME5, donde se solicita obtener la mediana para variables cuantitativas; es decir, encontrar un resumen estadístico de posición central para datos numéricos. Esta situación-problema se presenta en la actividad de la Figura 1.

En la Figura 1 se observa la SPMO1 donde se solicita la moda, es decir, el valor más frecuente de las estaturas de cada conjunto de datos. La SPMO2 se presenta en el inciso a) de la Figura 7, donde se solicita identificar la moda de un conjunto de datos cualitativos nominales.

3. Define la moda y lo que significa en cada caso.
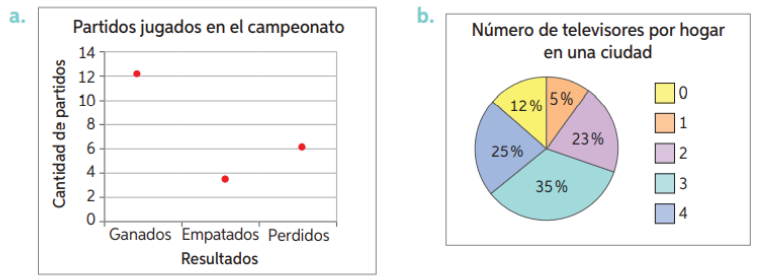

Figura 7. Situación-problema 2 asociada a la moda Nota. TE, p. 203.

Asimismo, en este estudio hemos considerado una nueva situación-problema asociada a la moda, SPMO3, donde se solicita obtener la moda de un listado de datos como tarea de tipo procedimental (ver Figura 8). 
1. Determina la moda de cada conjunto de datos.

a. $1,2,1,1,1,5,3,4,5,4,5,4,5,6,5,4,1,2,1,5,5,6,8$.

Figura 8. Situación-problema 3 asociada a la moda Nota. CA, p. 112

En la Tabla 3 se presenta un resumen de las situaciones-problema que se presentan en los textos escolares chilenos de séptimo básico, considerando como referencia aquellas que se citan en Cobo (2003) y Mayén (2009). Además, añadimos aquellas (letras cursivas) que surgen del análisis propio de este artículo. Como se puede observar, no se encontraron tres situaciones-problema asociadas a la media: SPM1, SPM2 y SPM4.

Tabla 3. Situaciones-problema que presentan los textos escolares de séptimo básico. Nota. Elaboración propia.

\begin{tabular}{|l|c|c|}
\hline Situación-problema & TE & CA \\
\hline $\begin{array}{l}\text { SPM1. Media, estimar una medida a partir de } \\
\text { diversas mediciones realizadas, en presencia } \\
\text { de errores }\end{array}$ & & \\
\hline $\begin{array}{l}\text { SPM2. Media, obtener una cantidad equitativa } \\
\text { al hacer un reparto }\end{array}$ & & \\
\hline $\begin{array}{l}\text { SPM3. Media, obtener un elemento } \\
\text { representativo en distribuciones } \\
\text { aproximadamente simétricas }\end{array}$ & $\mathrm{X}$ & $\mathrm{X}$ \\
\hline $\begin{array}{l}\text { SPM4. Media, como el valor más probable al } \\
\text { tomar un elemento al azar de un conjunto de } \\
\text { valores }\end{array}$ & & \\
\hline $\begin{array}{l}\text { SPM5. Media, para comparar dos } \\
\text { distribuciones de datos con variables } \\
\text { numéricas }\end{array}$ & $\mathrm{X}$ & $\mathrm{X}$ \\
\hline $\begin{array}{l}\text { SPM6. Media, obtener la media de un listado de } \\
\text { datos como ejercitación }\end{array}$ & & $\mathrm{X}$ \\
\hline $\begin{array}{l}\text { SPME1. Mediana, obtener la mediana cuando } \\
\text { la media no es representativa }\end{array}$ & $\mathrm{X}$ & \\
\hline $\begin{array}{l}\text { SPME2. Mediana, obtener la posición central } \\
\text { para variables ordinales }\end{array}$ & & $\mathrm{X}$ \\
\hline $\begin{array}{l}\text { SPME3. Mediana, para comparar conjuntos de } \\
\text { datos usando, o no, gráficos }\end{array}$ & $\mathrm{X}$ & \\
\hline $\begin{array}{l}\text { SPME4. Mediana, obtener la mediana de un } \\
\text { listado de datos como ejercitación }\end{array}$ & $\mathrm{X}$ \\
\hline $\begin{array}{l}\text { SPME5. Mediana, obtener la posición central } \\
\text { para variables cuantitativas }\end{array}$ & $\mathrm{X}$ \\
\hline $\begin{array}{l}\text { SPMO1. Moda, obtener el valor más frecuente } \\
\text { o dominante del conjunto de datos }\end{array}$ & $\mathrm{X}$ \\
\hline $\begin{array}{l}\text { SPMO2. Moda, obtener el valor representativo } \\
\text { en datos cualitativos }\end{array}$ & $\mathrm{X}$ & \\
\hline $\begin{array}{l}\text { SPMO3. Moda, obtener la moda de un listado } \\
\text { de datos como ejercitación }\end{array}$ & & $\mathrm{X}$ \\
\hline
\end{tabular}

\subsection{Procedimientos}

En segundo lugar, se analizaron los procedimientos, es decir, los algoritmos de cálculo que se requieren para realizar las diversas situaciones-problema.

Con relación a los procedimientos de cálculo para la media, encontramos cuatro de los siete considerados como referencia. Estos dependen de la forma de presentación de los datos: en un listado de datos (PM1, ver Figura 2) o en un gráfico (PM4, ver Figura 1); o bien, de lo que se solicita al estudiante: obtener un dato faltante a partir del valor de la media (PM6, ver Figura 9) o determinar una distribución de datos a partir del valor de la media (PM7, ver Figura 10).

2. Si la media aritmética de $10-15-12-X$ es 13 , ¿Cuál es el valor de $X$ ?
A. 10
B. 12
C. 13
D. 15

Figura 9. Procedimiento 6 para la media Nota. CA, p. 115.

4. Desafío Crea una tabla de frecuencia con un conjunto de 10 números que cumplan las siguientes condiciones:

a. $\bar{X}=45$, el valor mínimo es 10 , el rango es 90 y $\bar{X}>M e$.

b. $\bar{X}=116$, el valor máximo es 160 , el rango es 70 y $\bar{X}>M c$.

Figura 10. Procedimiento 7 para la media Nota. TE, p. 205.

Con respecto a los procedimientos de cálculo para la mediana, encontramos cuatro de los seis considerados como referencia; estos dependen de la presentación y número de datos. El PME1 y el PME2 se presentan en los incisos a) y c) de la actividad de la Figura 6, cálculo de la mediana para datos aislados con número de datos impar y par, respectivamente. El PM3 se muestra en la actividad de la Figura 11, cálculo de la mediana a partir de los datos presentados en una tabla de frecuencias, y el PME6 se muestra en la Figura 1, cálculo de la mediana a partir de los datos presentados en un gráfico.

\footnotetext{
Un curso realizó una campaña solidaria para reunir azúcar: 4 estudiantes llevaron $1 \mathrm{~kg}$, 11 llevaron $2 \mathrm{~kg}, 13$ llevaron $3 \mathrm{~kg}$ y 4 aportaron con $4 \mathrm{~kg}$. Identifica

Paso 1: Ordena los datos en una tabla de frecuencia y calcula la posición de la mediana. Como la cantidad de datos es par, entonces hay dos datos centrales, cuya posición está dada por: $\frac{\mathrm{n}}{2}=\frac{32}{2}=16 \quad$ Dato central 1

$\frac{\mathrm{n}}{2}+1=\frac{32}{2}+1=17 \quad$ Dato central 2

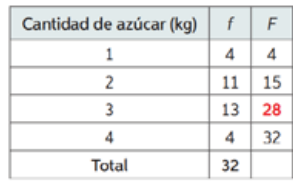

Paso 2: En $\mathrm{F}$ encuentra la posición 16 y 17 o que contiene a ambas. La frecuencia que las contiene es 28 , por lo que la mediana es 3 .

Paso 3: Interpreta la mediana. Que la mediana sea $3 \mathrm{~kg}$ significa que la mitad de los estudiantes que donaron la mayor cantidad de azúcar aportó como mínimo $3 \mathrm{~kg}$.
}

Figura 11. Procedimiento 3 para la mediana Nota. TE, p. 204. 
En relación con los procedimientos de cálculo para la moda, se identificaron tres de los cuatro considerados como referencia. Estos dependen de la presentación de los datos: a) listado de datos (PMO1, ver Figura 8), tabla de frecuencias (PMO2, ver el inciso $\mathrm{C}$ ) de la actividad de la Figura 12, donde solicita describir el cálculo de la moda a partir de la observación de los datos de una tabla) y gráfico (PMO4, ver Figura 1).

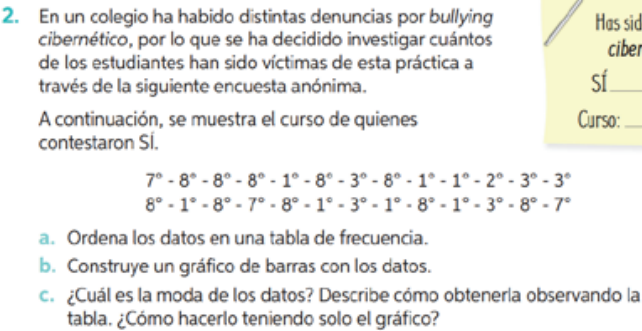

Figura 12. Procedimiento 2 para la moda Nota. TE, p. 203.

En la Tabla 4 se presenta un resumen de los procedimientos de cálculo de la media, mediana y moda que se incluyen, explícita o implícitamente, en los textos escolares de nuestra muestra.

Tabla 4. Procedimientos que presentan los textos escolares de séptimo básico. Nota. Elaboración propia.

\begin{tabular}{|c|c|c|}
\hline Procedimiento & TE & CA \\
\hline $\begin{array}{l}\text { PM1. Media, variable discreta o continua con datos } \\
\text { aislados }\end{array}$ & $x$ & $x$ \\
\hline \multicolumn{3}{|l|}{$\begin{array}{l}\text { PM2. Media, una variable discreta o continua con } \\
\text { datos presentados en tablas de frecuencias }\end{array}$} \\
\hline \multicolumn{3}{|l|}{$\begin{array}{l}\text { PM3. Media, variable continua o discreta con datos } \\
\text { agrupados en intervalos de clases }\end{array}$} \\
\hline PM4. Media, cálculo gráfico & $X$ & $X$ \\
\hline \multicolumn{3}{|l|}{ PM5. Media, cálculo con calculadora u ordenador } \\
\hline $\begin{array}{l}\text { PM6. Media, inversión del algoritmo de cálculo de la } \\
\text { media }\end{array}$ & $X$ & $X$ \\
\hline $\begin{array}{l}\text { PM7. Media, construir una distribución para una } \\
\text { media dada }\end{array}$ & $x$ & \\
\hline $\begin{array}{l}\text { PME1. Mediana, datos aislados (número de datos } \\
\text { impar) }\end{array}$ & $x$ & $x$ \\
\hline PME2. Mediana, datos aislados (número de datos par) & $x$ & $x$ \\
\hline $\begin{array}{l}\text { PME3. Mediana, datos en tabla de frecuencias } \\
\text { (número de datos par) }\end{array}$ & $x$ & \\
\hline \multicolumn{3}{|l|}{$\begin{array}{l}\text { PME4. Mediana, datos en tabla de frecuencias } \\
\text { (número de datos impar) }\end{array}$} \\
\hline \multicolumn{3}{|l|}{ PME5. Mediana, datos agrupados en clases } \\
\hline PME6. Mediana, cálculo gráfico & & $x$ \\
\hline $\begin{array}{l}\text { PMO1. Moda, variable discreta o cualitativa con datos } \\
\text { aislados }\end{array}$ & $X$ & $x$ \\
\hline $\begin{array}{l}\text { PMO2. Moda, variable discreta o cualitativa con datos } \\
\text { en una tabla de frecuencias }\end{array}$ & $X$ & \\
\hline \multicolumn{3}{|l|}{$\begin{array}{l}\text { PMO3. Moda, variable discreta con datos agrupados } \\
\text { en clases o variable continua }\end{array}$} \\
\hline PMO4. Moda, cálculo gráfico & $x$ & $x$ \\
\hline
\end{tabular}

\subsection{Conceptos-definición}

En tercer lugar, se analizó el concepto-definición de las MTC en la muestra de textos escolares. En el libro del estudiante encontramos expresado explícitamente un concepto-definición para cada MTC: a) CDM2, la media como promedio aritmético de un conjunto de datos (ver Figura 13); b) CDME1, la mediana como centro o valor central de una distribución de datos (ver Figura 14), y c) CDMO1, la moda como el valor más frecuente de la variable (ver Figura 15).

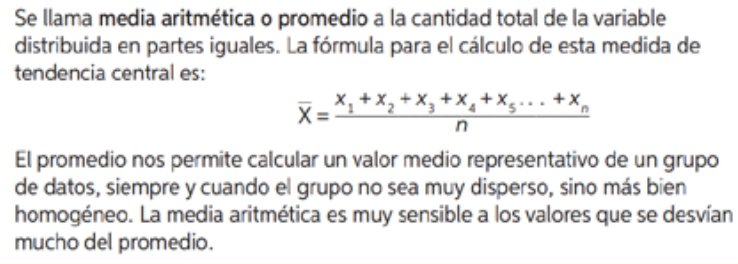

$$
\overline{\mathrm{X}}=\frac{x_{1}+x_{2}+x_{3}+x_{4}+x_{5} \ldots+x_{n}}{n}
$$

El promedio nos permite calcular un valor medio representativo de un grupo de datos, siempre y cuando el grupo no sea muy disperso, sino más bien homogéneo. La media aritmética es muy sensible a los valores que se desvian mucho del promedio.

Figura 13. Concepto-definición 2 de la media Nota. TE, p. 199

La mediana corresponde al valor que ocupa el término central de un conjunto de datos una vez ordenados de menor a mayor o viceversa. Cuando la cantidad de datos ( $n$ ) de un conjunto es par, la mediana corresponde a la media aritmética de los dos términos centrales una vez que estos se ordenan.

Figura 14. Concepto-definición 1 de la mediana Nota. TE, p. 203.

Se llama moda (Mo) de un conjunto de datos a la variable que presenta mayor tendencia de ocurrencia. Para calcular esta medida de tendencia central, identificamos la variable cuya frecuencia absoluta es mayor que el resto de los datos.

Un conjunto de datos puede tener más de una moda, o bien puede que no exista moda (amodal) si todos los datos se distribuyen con la misma frecuencia.

Figura 15. Concepto-definición 1 de la moda Nota. TE, p. 202.

Además, se analizó el uso implícito que se hace de cada concepto-definición de las MTC para la solución de las situaciones-problema. A partir de esto, encontramos aquellos que no aparecen de forma explícita, sino como situaciones-problema resueltas o propuestas a los estudiantes: a) CDM1, la media como la suma ponderada de cada uno de los valores de la variable (o bien, cada uno de los valores "medios" de los intervalos de clase) multiplicado por su frecuencia, es decir, como algoritmo de cálculo (ver Figura 3); CDME2, la mediana como valor de la variable que divide en dos partes iguales a un conjunto de datos ordenados (ver paso 3 de la actividad de la Figura 11), y CDMO2, la moda como el valor que corresponde al máximo del gráfico de barras, histograma o diagrama de puntos (ver Figura 1). 
En la Tabla 5 presentamos los conceptos-definición de las MTC que se han mencionado, así como la presencia de estas en cada uno de los textos escolares de séptimo básico analizados.

Tabla 5. Conceptos-definición que presentan los textos escolares de séptimo básico. Nota. Elaboración propia.

\begin{tabular}{|l|l|l|}
\hline Concepto-definición & TE & CA \\
\hline CDM1. Media, como algoritmo & & $X$ \\
\hline CDM2. Media, como promedio & $X$ & \\
\hline $\begin{array}{l}\text { CDME1. Mediana, centro de la distribución } \\
\text { (valor central) }\end{array}$ & $X$ & \\
\hline CDME2. Mediana, divide en dos partes iguales & $X$ & $X$ \\
\hline $\begin{array}{l}\text { CDME3. Mediana en un diagrama acumulativo } \\
\text { es n/2 }\end{array}$ & & \\
\hline $\begin{array}{l}\text { CDME4. Mediana en una distribución empírica } \\
\text { es } 1 / 2\end{array}$ & & \\
\hline CDMO1. Moda, valor más frecuente & $X$ & \\
\hline CDMO2. Moda, valor máximo en un gráfico & & $X$ \\
\hline
\end{tabular}

\subsection{Propiedades}

Posteriormente, se analizaron las propiedades introducidas de forma explícita o implícita, clasificándolas en numéricas, algebraicas y estadísticas. Con respecto a las propiedades, reconocemos que son igual de relevantes que los conceptos-definición para el estudio y comprensión de la media, mediana y moda. No obstante, su tratamiento en los textos escolares de séptimo básico es muy inferior, ya que hemos identificado pocas: tres numéricas, una algebraica y cinco estadísticas. Por ejemplo, la PN2 (la mediana y la media pueden no coincidir con ninguno de los valores de los datos, mientras que la moda siempre es uno de estos valores) y la PN3 (para el cálculo de la media se tienen en cuenta todos valores de los datos, pero no en la mediana y la moda) se presentan, de forma implícita, en el enunciado de la Figura 16.

\footnotetext{
En este caso, la cantidad de datos sería par (16 jóvenes), por lo tanto, al ordenar los datos de menor a mayor existen dos términos centrales. En estas situaciones, cuando n es par, la mediana es la media aritmética de los dos términos centrales y no necesariamente este valor pertenece al conjunto de datos.
}

\section{Figura 16. Propiedades numéricas 2 y 3} Nota. Elaboración propia.

En el libro del estudiante encontramos, en el segundo párrafo de la Figura 15, la propiedad algebraica PA7: la moda puede o no existir, mientras que la media y la mediana siempre existen. Con respecto a las propiedades estadísticas presentes en los textos escolares analizados, a continuación, ejemplificaremos tres de las cinco identificadas: PE2, la media coincide con el centro del conjunto de datos; PE3, la media, mediana y moda coinciden en distribuciones simétricas, y PE4, la media es sensible a la variación de los datos del conjunto, la mediana y la moda no, se presentan implícitamente en las Figuras 17,18 y 13 , respectivamente.

Las medidas de dispersión son valores numéricos que permiten medir qué tan dispersos están los datos alrededor de un valor central, por lo general la media.

\section{Figura 17. Propiedad estadística 2} Nota. TE, p. 200

$\rightarrow$ Cuando los datos sean simétricos, la media y la mediana serán esencialmente el mismo número.

Figura 18. Propiedad estadística 3 Nota. TE, p. 207.

En la Tabla 6 se presentan las propiedades numéricas, algebraicas y estadísticas de cada una de las MTC, y se señalan aquellas que se identificaron en los libros analizados.

Tabla 6. Propiedades que presentan los textos escolares de séptimo básico. Nota. Elaboración propia.

\begin{tabular}{|c|c|c|}
\hline $\begin{array}{l}\text { Propiedades numéricas }(N) \text {, algebraicas }(A) \text { y } \\
\text { estadísticas }(E)\end{array}$ & TE & CA \\
\hline $\begin{array}{l}\text { PN1. Media, mediana y moda pertenecen al } \\
\text { rango de la variable }\end{array}$ & & \\
\hline $\begin{array}{l}\text { PN2. La moda coincide con algún dato de la } \\
\text { distribución, la mediana en algunos casos, } \\
\text { pero la media no }\end{array}$ & $X$ & $X$ \\
\hline $\begin{array}{l}\text { PN3. Para la media y la moda se tienen en } \\
\text { cuenta todos los valores del conjunto, pero no } \\
\text { en la mediana }\end{array}$ & $X$ & \\
\hline $\begin{array}{l}\text { PN4. El valor de la media cambia cuando } \\
\text { se modifica cualquier dato del conjunto; } \\
\text { en cambio la moda y la mediana son, en } \\
\text { ocasiones, invariantes }\end{array}$ & $X$ & $X$ \\
\hline $\begin{array}{l}\text { PA1. La moda es una operación interna, } \\
\text { mientras que la media y la mediana no }\end{array}$ & & \\
\hline $\begin{array}{l}\text { PA2. La media, mediana y moda no tienen } \\
\text { elemento neutro ni simétrico }\end{array}$ & & \\
\hline PA3. No tienen propiedad asociativa & & \\
\hline PA4. Son operaciones conmutativas & & \\
\hline PA5. Conservan cambios de origen y escala & & \\
\hline $\begin{array}{l}\text { PA6. La media de la suma de dos o más } \\
\text { variables es la suma de la media de dichas } \\
\text { variables. En el caso de la mediana y la moda } \\
\text { no se cumple }\end{array}$ & & \\
\hline $\begin{array}{l}\text { PA7. La moda puede o no existir, mientras que } \\
\text { la media y la mediana siempre existen }\end{array}$ & $X$ & \\
\hline $\begin{array}{l}\text { PE1. La media, mediana y moda son } \\
\text { representantes de un colectivo }\end{array}$ & $X$ & \\
\hline $\begin{array}{l}\text { PE2. La media coincide con el centro del } \\
\text { conjunto de datos }\end{array}$ & $X$ & \\
\hline
\end{tabular}




\begin{tabular}{|l|l|l|}
\hline $\begin{array}{l}\text { PE3. Media, mediana y moda coinciden en } \\
\text { distribuciones simétricas }\end{array}$ & $X$ & \\
\hline $\begin{array}{l}\text { PE4. La media es sensible a la variación de los } \\
\text { datos del conjunto, la mediana y la moda no }\end{array}$ & $X$ & \\
\hline $\begin{array}{l}\text { PE5. La suma de las desviaciones del conjunto } \\
\text { de datos con respecto a su media es cero }\end{array}$ & & \\
\hline $\begin{array}{l}\text { PE6. Respecto a la media, la suma de } \\
\text { cuadrados de las desviaciones es mínima }\end{array}$ & & \\
\hline $\begin{array}{l}\text { PE7. Es preferible la mediana a la media en } \\
\text { datos agrupados en intervalos, en los que al } \\
\text { menos uno es abierto }\end{array}$ & & \\
\hline $\begin{array}{l}\text { PE8. Existe moda para variables cualitativas y } \\
\text { cuantitativas }\end{array}$ & $X$ & \\
\hline $\begin{array}{l}\text { PE9. La mediana es mejor representante que la } \\
\text { media en distribuciones no unimodales }\end{array}$ & & \\
\hline
\end{tabular}

\subsection{Elementos lingüísticos}

En quinto lugar, analizamos los elementos lingüísticos. Se consideraron todos aquellos términos, palabras, expresiones, símbolos, tablas y gráficos ligados a las MTC, que sirven para enunciar los conceptosdefinición y propiedades de dicho objeto, así como para describir las situaciones-problema y representar los datos.

En la Tabla 7 se presentan los elementos lingüísticos y se señalan aquellos que se identificaron en los libros analizados. En general, los elementos lingüísticos utilizados en nuestra muestra de textos escolares son similares a los resultados reportados en Cobo (2003) y Mayén (2009).

Tabla 7. Elementos lingüísticos que presentan los textos escolares de séptimo básico. Nota. Elaboración propia.

\begin{tabular}{|l|l|l|}
\hline Elemento lingüístico & TE & CA \\
\hline Términos & $\mathrm{X}$ & $\mathrm{X}$ \\
\hline Símbolos & $\mathrm{X}$ & $\mathrm{X}$ \\
\hline Tablas & $\mathrm{X}$ & $\mathrm{X}$ \\
\hline Gráficos & $\mathrm{X}$ & $\mathrm{X}$ \\
\hline
\end{tabular}

\subsection{Argumentos}

Con respecto a los argumentos que se usan para justificar los procedimientos, propiedades y conceptos-definición, se encontraron ARG1 y ARG4 (ver Tabla 8), referentes a la justificación de ejemplos o contraejemplos, y razonamientos verbales deductivos, respectivamente. Estos se muestran en la Figura 11 que corresponde a un ejemplo de la mediana, en donde se explica su resolución y se presenta un argumento que justifica su resultado y su interpretación.
Tabla 8. Argumentos que presentan los textos escolares de séptimo básico. Nota. Elaboración propia.

\begin{tabular}{|l|l|l|}
\hline Argumento & TE & CA \\
\hline $\begin{array}{l}\text { ARG1. Comprobación de casos particulares y } \\
\text { contraejemplos }\end{array}$ & $X$ & \\
\hline ARG2. Uso de gráficos como justificación & & \\
\hline ARG3. Razonamiento algebraico & & \\
\hline ARG4. Razonamiento verbal deductivo & $X$ & \\
\hline
\end{tabular}

\section{Conclusiones}

Las medidas de tendencia central (media, mediana y moda) son generalmente empleadas para resumir un conjunto de datos, he ahí su importancia como parte de la alfabetización estadística de cualquier ciudadano del mundo, pues estas permiten generar conclusiones, inferencias, entre otros. Así que, en este estudio, al mirar las actividades presentes en los textos escolares chilenos de séptimo básico, asumíamos que este documento representa la visión que se desarrolla alrededor de estas nociones en dicho grado escolar, la cual describiremos enseguida. Para ello, se miraron los seis elementos que describen el significado de un objeto matemático, a saber, situaciones-problema, procedimientos, conceptos-definición, propiedades, elementos lingüísticos y argumentos.

Uno de los elementos que más nos llamó la atención es que únicamente tres situaciones-problema asociadas a las MTC se comparten en ambos textos escolares: a) SPM3, la media para obtener un elemento representativo en distribuciones aproximadamente simétricas; b) SPM5, la media para comparar dos distribuciones de datos con variables numérica, y c) SPMO, la moda para obtener el valor más frecuente o dominante del conjunto de datos. Mientras, las otras situaciones-problema no se presentan (por ejemplo: SPM1, la media para estimar una medida a partir de diversas mediciones realizadas, en presencia de errores; SPM2, la media para obtener una cantidad equitativa al hacer un reparto, y SPM4, la media como el valor más probable al tomar un elemento al azar de un conjunto de valores), o bien, solo se encuentran presentes en uno de los textos escolares (por ejemplo: SPM6, obtener la media de un listado de datos como ejercitación, y SPME1, obtener la mediana cuando la media no es representativa). Esto lo percibimos como una dificultad u obstáculo para la comprensión significativa de las MTC. En comparación con estudios previos, SPM1 y SMP2 son poco usuales en los libros de textos españoles (Cobo, 2003) y mexicanos (Mayén, 2009); mientras que SPM4 tampoco se presenta en dichos estudios.

En función a los procedimientos de cálculo de las MTC que se presentan en los textos escolares analizados, identificamos que se promueven la mayoría de estos, a excepción de PM2, asociado al cálculo de la 
media de una variable discreta o continua con datos presentados en tablas de frecuencias; PM3, asociado al cálculo de la media de una variable continua o discreta con datos agrupados en intervalos de clases; PM5, asociado al cálculo de la media mediante el uso de una calculadora u ordenador; PME4, asociado al cálculo de la mediana de datos en tabla de frecuencias (número de datos impar); PME5, asociado al cálculo de la mediana de datos agrupados en clases; y PMO3, asociado al cálculo de la moda de una variable discreta con datos agrupados en clases o variable continua. En relación con los estudios previos, nuestros resultados coinciden en la carencia de PME4 con los textos escolares españoles (Cobo, 2003) y de PM5 y PME5 con los mexicanos (Mayén, 2009).

Con respecto a los conceptos-definición identificados en este estudio, tres se presentan de forma explícita en el texto del estudiante (CDM2, la media como promedio; CDME1, la mediana como centro de la distribución o valor central, y CDMO1, la moda como valor más frecuente) y tres de manera implícita en el cuaderno de actividades (CDM1, la media como algoritmo; CDME2, la mediana divide en dos partes iguales al conjunto de datos, y CDMO2, la moda como valor máximo en un gráfico). Estos conceptosdefinición son semejantes a los encontrados en el estudio de Cobo (2003) y Mayén (2009), la única diferencia con los libros de texto mexicanos es que encontramos aquel que indica obtener la moda a partir de la lectura de un gráfico estadístico.

Dentro de las propiedades se identificó que en los textos escolares solo se presentan tres numéricas (PN2, la moda coincide con algún dato de la distribución, la mediana en algunos casos, pero media no; PN3, para la media y moda se tienen en cuenta todos los valores del conjunto, pero no en mediana; y PN4, el valor de la media cambia cuando se modifica cualquier dato del conjunto, en cambio la moda y la mediana son, en ocasiones, invariantes), una algebraica (PA7, la moda puede o no existir, mientras que la media y la mediana siempre existen) y cinco estadísticas (PE1, la media, mediana y moda son representantes de un colectivo; PE2, la media coincide con el centro del conjunto de datos; PE3, la media, mediana y moda coinciden en distribuciones simétricas; PE4, la media es sensible a la variación de los datos del conjunto, la mediana y la moda no, y PE8, existe moda para variables cualitativas y cuantitativas). Estas propiedades se presentan en mayor medida en los textos españoles (Cobo, 2003) que en los mexicanos (Mayén, 2009) analizados en estudios previos.

En relación con los elementos lingüísticos, estos muestran los cuatro tipos: términos, símbolos, tablas y gráficos. En general, estos elementos también se presentan en los resultados de los estudios de Cobo (2003) y Mayén (2009). En el caso de los argumentos asociados a las MTC, se presentan dos: comprobación de casos particulares y contraejemplos, y razonamientos verbales deductivos; mismos que se presentan en la mayoría de los textos españoles y mexicanos analizados por Cobo (2003) y Mayén (2009), respectivamente.

En la Figura 19 se presenta un hexágono que representa el significado ideal (puntos amarillos) apoyado en las seis categorías propuestas para su análisis. Los hexágonos de líneas continuas (azul y anaranjado) corresponden a la proporción del total de categorías para cada elemento de significado encontrada en el texto del estudiante (TE) y el cuaderno de actividades (CA), respectivamente. Como se puede evidenciar en esta representación, los textos escolares de séptimo básico se encuentran carentes de algunos elementos; esto implica que la comprensión que el estudiante obtenga de las MTC se encuentre posiblemente limitada.

En concreto, principalmente hay una pobreza con respecto al tipo de situaciones-problema y conceptos-definición, además de que ambos textos no comparten o promueven los mismos elementos, ni con la misma proporción; esto por las características propias de cada texto escolar. En general, la forma en la que se presentan las actividades en los libros no propicia una comprensión apropiada de las MTC, siendo solo el uso de elementos lingüísticos que se presenta desarrollado en su totalidad.

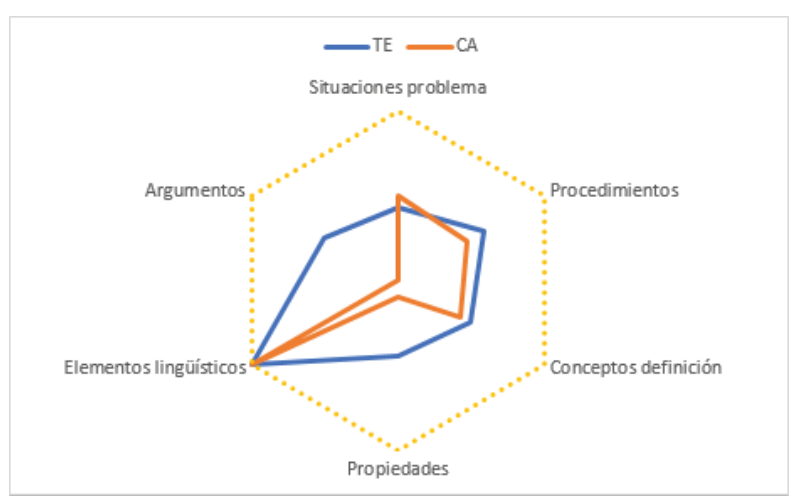

Figura 19. Distribución general de los elementos del significado de las MTC . Nota. Elaboración propia.

Con este estudio, a partir del análisis de la presencia de cada una de las categorías asociadas a cada uno de los elementos del significado de la media, mediana y moda en los textos escolares de séptimo básico, se muestra una aproximación a la forma en que estas se trabajan en las aulas chilenas. Con esta información, se recomienda diseñar una propuesta didáctica donde se aborden las diversas situaciones-problema, procedimientos, conceptos-definición, propiedades, elementos lingüísticos y argumentos de las MTC, que consideramos necesarios para su incorporación en las aulas para lograr su enseñanza más significativa. En concreto, se sugiere contextualizar los diferentes elementos de significado en actividades relacionadas con estudiantes de séptimo básico en orden de dificultad progresiva con base en el tiempo disponible. 


\section{Agradecimientos}

Este trabajo ha sido desarrollado en el marco del Proyecto Fondecyt 1200005 financiado por la Agencia

Nacional de Investigación y Desarrollo (ANID) de Chile. 


\section{Referencias}

Batanero, C. (2000). Significado y comprensión de las medidas de posición central. Uno. Revista de Didáctica de las Matemáticas, 25, 41-58. https://www. ugr.es/ batanero/pages/ARTICULOS/isboa.pdf

Cabrera, B. (2014). Contenido de la media aritmética en los libros de texto y su influencia en la comprensión por estudiantes del primer ciclo de la Universidad Nacional Micaela Bastidas de Apurímac. En N. Rubio (Ed.), VII Coloquio Internacional Enseñanza de las Matemáticas. Educación Matemática en contexto (11731175). Pontificia Universidad Católica del Perú.

Cantoral, R., Montiel, G., y Reyes-Gasperini, D. (2015). Análisis del discurso matemático escolar en los libros de texto, una mirada desde la teoría socioepistemológica. Avances de Investigación en Educación Matemática, 8, 9-28. https://doi.org/10.35763/aiem.v1i8.123

Carvalho, J., y Gitirana, V. (2014). Média aritmética - uma análise das atividades do livro didáctico de matemática adotados no brasil. En P. Lestón (Ed.), Acta Latinoamericana de Matemática Educativa 27 (pp. 681-688). Comité Latinoamericano de Matemática Educativa.

Chevallard, Y. (1991). La transposición didáctica. Del saber sabio al saber enseñado. Aique.

Cobo, B. (2003). Significados de las medidas de posición central para los estudiantes de secundaria [Tesis doctoral, Universidad de Granada]. https://www. ugr.es/ batanero/pages/ARTICULOS/tesiscobo.pdf

Cobo, B., y Batanero, C. (2004). Significado de la media en los libros de texto de secundaria. Enseñanza de las Ciencias, 22(1), 5-18. https://doi.org/10.5565/rev/ ensciencias.3899

Del Pino, G., y Estrella, S. (2012). Educación estadística: relaciones con la matemática. Pensamiento Educativo, 49(1), 53-64. http://ojs.uc.cl/index.php/pel/article/ view/25747/20671

Del Puerto, S., Seminara, S., y Minnaard, C. (2007). Identificación y análisis de los errores cometidos por los alumnos en Estadística Descriptiva. Revista iberoamericana de educación, 43(3), 1-8. https://rieoei. org/RIE/article/view/2331

Díaz-Levicoy, D., Morales-García, L., y Rodríguez-Alveal, F. (2020). Las medidas de tendencia central en libros de texto de Educación Primaria en México. Revista Paradigma, 41, 706-729. https://doi.org/10.37618/ PARADIGMA.1011-2251.2020.p706-729.id819
Escobedo, J. M., y Mayén, S. (2018). Evolución en la comprensión de estudiantes de telebachillerato de un problema de estimación de media y mediana a partir de un gráfico. En J. D. Zacarías, H. Cruz, F. Velasco, B. Juárez, V. H. Vázquez, H. Reyes y F. Tajonar (Eds.), Actualidad en la Educación Estadística y Probabilística (pp. 111-137). Benemérita Universidad Autónoma de Puebla.

Estrella, S. (2008). Medidas de tendencia central en la enseñanza básica en Chile. Revista Chilena de Educación Matemática, 4(1), 20-32. http://static.ima.ucv.cl.s3. amazonaws.com/wp-content/uploads/2015/05/ RECHIEM_TD_Estrella-2008-con-tapa.pdf

Estrella, S. (2016). Comprensión de la media por profesores de educación primaria en formación continua. Revista electrónica de investigación educativa, 18(1), 13-22. https://redie.uabc. $\mathrm{mx} /$ redie/article/ view/635

Font, V., Godino, J. D., y D'Amore, B. (2007). An ontosemiotic approach to representations in mathematics education. For the Learning of Mathematics, 27(2), 2-7. http://www.dm.unibo.it/rsddm/ it/articoli/damore/617\%20\%202007\%20FLM.pdf

Font, V., Godino, J. D., y Gallardo, J. (2013). The emergence of objects from mathematical practices. Educational Studies in Mathematics, 82, 97-124. https:// doi.org/10.1007/s10649-012-9411-0

Gal, I. (2004). Statistical Literacy, Meanings, Components, Responsibilities. En D. Ben-Zvi y J. Garfield (Eds.), The Challenge of Developing Statistical Literacy, Reasoning and Thinking (pp. 47-78). Kluwer Academic Publishers. https://doi.org/10.1007/1-40202278-6_3

Godino, J. D., y Batanero, C. (1994). Significado institucional y personal de los objetos matemáticos. Recherches en Didactique des Mathématiques, 14(3), 325-355. https://www.ugr.es/ jgodino/funcionessemioticas/03_SignificadosIP_RDM94.pdf

Godino, J. D., Batanero, C., y Font, V. (2007). The onto-semiotic approach to research in mathematics education. ZDM. The International Journal on Mathematics Education, 39(1-2), 127-135. https://doi. org/10.1007/s11858-006-0004-1

Godino, J. D., Batanero, C., y Font, V. (2020). El enfoque ontosemiótico: implicaciones sobre el carácter prescriptivo de la didáctica. Revista Chilena De Educación Matemática, 12(2), 47-59. https://doi. org/10.46219/rechiem.v12i2.25

Herbel, B. A. (2007). From intended curriculum to written curriculum: Examining the "voice" of a mathematics textbook. Journal for Research in Mathematics Education, 38(4), 344-369. https://www. jstor.org/stable/30034878?seq=1 
Hernández, R., Fernández, C., y Baptista, P. (2010). Metodología de la investigación. McGraw Hill.

López-Noguero, F. (2002). El análisis de contenido como método de investigación. XXI. Revista de Educación, 4, 167-180. http://rabida.uhu.es/dspace/ handle/10272/1912

Mayén, S. (2009). Comprensión de las medidas de tendencia central por estudiantes mexicanos de Educación Secundaria y Bachillerato [Tesis doctoral, Universidad de Granada]. Repositorio Institucional de la Universidad de Granada. https://digibug.ugr.es/ handle/10481/2418

Mayén, S., Cobo, B., Batanero, C., y Balderas, P. (2007). Comprensión de las medidas de posición central en estudiantes mexicanos de bachillerato. Unión. Revista Iberoamericana de Educación Matemática, 9, 187-201. http://www.fisem.org/www/union/revistas/2007/9/ Union_009_016.pdf

Ministerio de Educación de Chile. (2016). Bases Curriculares. $7^{\circ}$ básico a $2^{\circ}$ medio. Autor.

Ministerio de Educación de Chile. (2018). Bases Curriculares. Primero a Sexto Básico. Autor.

Ocoró, L. V., y Ocoró, S. (2016). Análisis de las medidas de tendencia central en dos libros de textos escolares de grado séptimo: el caso de la media aritmética. En I. Álvarez y C. Sua (Eds.), Memorias del II Encuentro Colombiano de Educación Estocástica (184-190). Asociación Colombiana de Educación Estocástica.

Pérez-Serrano, G. (1994). Investigación cualitativa. Retos e interrogantes. I. Métodos. La Muralla.

Pino-Fan, L. (2013). Evaluación de la faceta epistémica del conocimiento didáctico-matemático de futuros profesores de bachillerato sobre la derivada [Tesis doctoral, Universidad de Granada]. https://www.ugr. es/ jgodino/Tesis_doctorales/Luis_Pino_tesis.pdf

Vásquez, C., y Alsina, Á. (2014). Enseñanza de la Probabilidad en educación primaria. Un desafío para la formación inicial y continua del profesorado. Números. Revista de Didáctica de las Matemáticas, 85, 5-23. http://www.sinewton.org/numeros/numeros/85/ Articulos_01.pdf 